1 Universidade Estadual do Pará (Uepa) - Belém (PA), Brasil.

suenf2007@gmail.com

2 Universidade Federal do Pará (UFPA) - Belém (PA), Brasil.

\section{Amandaba no Caeté: círculos de cultura como prática educativa no autocuidado de portadores de diabetes}

\author{
Amandaba in the Caeté: culture circles as an educational practice in \\ the self-care of patients with diabetes
}

Suelen Trindade Correa', Socorro Castelo-Branco ${ }^{2}$

DOI: 10.1590/0103-1104201912310

\begin{abstract}
RESUMO Objetivou-se avaliar a estratégia pedagógica dos círculos de cultura de Paulo Freire na adesão ao autocuidado em pacientes com Diabetes Mellitus. Foi realizado um ensaio clínico randomizado com 72 participantes, alocados em Grupo Intervenção (GI) e Grupo Controle (GC). O GI participou de seis círculos para problematizar e desvelar os temas geradores: alimentação, medicação, complicações do diabetes e cuidados com os pés; o GC participou apenas de consultas de rotina. Foi avaliada a mudança proporcionada pelos círculos após três meses de intervenção, por meio do Questionário de Atividades de Autocuidado com o Diabetes, glicemia capilar em jejum, pressão arterial sistólica e diastólica, Índice de Massa Corpórea e circunferência abdominal. O GI apresentou aumento estatisticamente significante na adesão ao autocuidado à alimentação saudável, prática de exercício físico, monitoramento glicêmico e

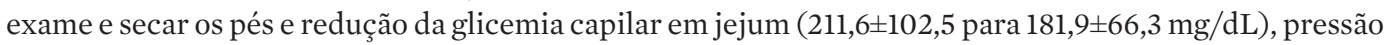
arterial sistólica de 125(120-140) para 120(117,5-130 mmHg) e circunferência abdominal (96,3 $\pm 9,5$ para $95,5 \pm 9,1 \mathrm{~cm}$ ), enquanto no GC não houve modificação durante o estudo. Os círculos de cultura, como abordagem educativa, foram capazes de melhorar as práticas de adesão ao autocuidado dos pacientes com diabetes e proporcionar a melhora em alguns parâmetros de risco cardiovascular.
\end{abstract}

PALAVRAS-CHAVE Educação em saúde. Diabetes Mellitus. Autocuidado. Estratégia Saúde da Família.

\begin{abstract}
The aim of this study was to evaluate the pedagogical strategy of Paulo Freire's culture circles in adherence to self-care in patients with Diabetes Mellitus. A randomized clinical test was performed with 72 participants, allocated into Intervention Group (IG) and Control Group (CG).The IG participated in six circles to discuss and unveil the generative themes: diet, medication, diabetes complications and foot care; the CG participated only in routine consultations. The change provided by the circles after three months of intervention was evaluated through the Diabetes Self-Care Activity Questionnaire, fasting capillary glycemia, systolic and diastolic arterial pressure, body mass index and abdominal circumference. The IG showed a statistically significant increase in self-care adherence to healthy diet, physical exercise, glycemic monitoring and examination and drying of the feet and reduced fasting capillary glycemia (211.6 \pm 102.5 to $181.9 \pm$ $66.3 \mathrm{mg} / \mathrm{dL}$ ), systolic arterial pressure 125(120-140) to $120(117.5-130 \mathrm{mmHg}$ ) and abdominal circumference (96.3 \pm 9.5 to $95.5 \pm 9.1 \mathrm{~cm})$, while in the CG there were no changes during the study. The culture circles, as an educational approach, were able to improve the self-care adherence practices of patients with diabetes and provide improvement in some cardiovascular risk parameters.
\end{abstract}

KEYWORDS Health education. Diabetes Mellitus. Self care. Family Health Strategy. 


\section{Introdução}

O Diabetes Mellitus é uma das condições crônicas mais emergentes do século XXI, e suas complicações são as principais causas de morte em muitos países'. Devido à cronicidade e à gravidade das complicações, é considerada uma doença onerosa, tanto para o indivíduo e sua família quanto para os serviços de saúde 2 .

A estimativa de pessoas no mundo com diabetes, em 2015, foi de 415 milhões, enquanto no Brasil tal estimativa correspondeu a 14,3 milhões $(9,4 \%)$ de brasileiros com a condição crônical. No Estado do Pará, a proporção de pessoas a partir de 18 anos que referiram ter diabetes foi de 3,8\%3; em Belém, os dados do Vigitel (2016) mostram uma proporção de 6,6\% $\mathbf{4}^{4}$.

O diabetes, sendo uma doença crônica que requer cuidados contínuos e permanentes com a saúde, que vão além do tratamento clínico, exige adoção, pelo paciente, de mudanças no estilo de vida e desenvolvimento de habilidades de autocuidado para que haja um controle da doença ${ }^{5}$.

A educação em saúde, sendo uma tecnologia leve ${ }^{6}$ que propicia conhecimentos, habilidades e capacidades indispensáveis para o autocuidado das pessoas com diabetes e sua família, ajuda os pacientes a tomar decisões, a autogerenciar a doença, a resolver problemas e a colaborar ativamente com a equipe de saúde no controle clínico e na qualidade de vida ${ }^{7}$.

Uma das abordagens de educação em saúde, utilizada na área da saúde desde a década de 1970, é o Círculo ('Amandaba' em TupiGuarani) de Cultura, modelo pedagógico do educador Paulo Freire ${ }^{8}$. Nele, o ambiente é diferente do da sala de aula tradicional, em que os educandos, ao ficarem dispostos em roda, favorecendo a interação e o diálogo entre si, irão aprender e ensinar mutuamente ${ }^{9}$.

Nesse modelo, que utiliza a problematização, a peça fundamental é o diálogo que procura aproximar os sujeitos, promovendo a reflexão crítica, a autonomia e o empoderamento deles, buscando, coletivamente, melhorar a compreensão da realidade e transformá-la ${ }^{10,11}$.
Vale lembrar que, em 2013, a Portaria ${ }^{\circ}$ 276 instituiu a Política Nacional de Educação Popular em Saúde, propondo práticas político-pedagógicas que transversalizem ações voltadas para a promoção, a proteção e a recuperação da saúde, considerando princípios como o diálogo, a amorosidade, a problematização, a construção partilhada do conhecimento, a emancipação e o compromisso com a construção de um projeto democrático e popular ${ }^{12}$. Assim, o Círculo de Cultura é uma metodologia com caráter democrático e libertador, cuja aprendizagem integral rompe com a fragmentação da realidade, estimulando a horizontalidade do educador com o educando, com valorização da cultura e do ser humano, favorecendo o senso crítico, a resolução de problemas e a transformação da realidade? .

O Círculo de Cultura, segundo Heidemann; Brandão ${ }^{9}$; Freire ${ }^{10}$, é desenvolvido nas seguintes fases: levantamento do universo vocabular/ levantamento temático, realizado por meio de encontros informais, entrevistas e/ou observações da realidade pelo coordenador do círculo, que levanta os vocábulos, as palavras, os temas de conteúdo emocional, as falas típicas dos educandos; escolha das palavras/temas geradores que são selecionados pelo coordenador do círculo, a partir do universo vocabular do grupo e que implique a realidade social, cultural, política etc. do educando, que são apresentados nos círculos para debate; problematização das situações vividas pelo grupo, por meio da codificação e descodificação dos temas geradores debatidos pelos educandos dentro de círculos (para produzir os debates, utilizam-se as fichas de cultura, que são desenhos/imagens relacionados aos temas geradores); desvelamento crítico, que objetiva a tomada de consciência da situação vivida e que, mediante a visão crítica, transforma a sua realidade.

Desse modo, a educação em saúde, voltada para o cuidado com o diabetes, realizada nessa perspectiva dialogal, reflexiva e crítica, poderá ser instrumento efetivo para a formação de um conhecimento crítico, ampliando a compreensão do paciente e a sua autonomia diante 
das condições de vida e sobre o diabetes ${ }^{\mathbf{1 3}}$. Portanto, o presente estudo teve como objetivo avaliar a estratégia pedagógica dos Círculos de Cultura na adesão ao autocuidado em pacientes com Diabetes Mellitus.

\section{Material e métodos}

Ensaio clínico randomizado desenvolvido com usuários portadores de Diabetes Mellitus acompanhados nas unidades de Estratégias Saúde da Família (ESF), na zona urbana do município de Bragança (PA), na Amazônia Brasileira, situada à margem do rio Caeté ou Caité ('mato bom' na língua Tupi).

O estudo foi aprovado pelo Comitê de Ética em Pesquisa do Instituto de Ciências da Saúde, da Universidade Federal do Pará, com o parecer CAEE 57049416.9.0000.0018, e orientou-se pelos princípios éticos da Resolução ${ }^{\circ}$ $466 / 2012$. Todos os participantes assinaram o Termo de Consentimento Livre e Esclarecido após receberem explicação verbal e escrita sobre o objetivo do estudo, sobre os procedimentos a serem realizados, sobre os riscos e os benefícios de sua participação.

A amostra foi calculada com base na população de 30 anos a 60 anos (de ambos os gêneros), sendo sorteadas quatro unidades $(n=4)$ para o Grupo Controle (GC) e quatro unidades $(n=4)$ para o Grupo Intervenção (GI). Assim, a amostra calculada seria de 244 usuários com diabetes pertencentes a elas, que foram convidados. No entanto, 166 pessoas recusaram a participação em virtude da concomitância das reuniões com a jornada de trabalho. Foram ainda excluídos dois usuários por apresentarem nefropatia, dois por neuropatia com dificuldade de locomoção e três por retinopatia, com baixa acuidade visual.

Aos participantes do GC, foram oferecidas as ações de rotina das unidades, como: consulta médica e orientações individuais dos profissionais que trabalham nas unidades. Os participantes do GI foram submetidos às atividades de educação em saúde baseada nos
Círculos de Cultura de Paulo Freire e orientados a continuarem o atendimento individual na unidade, como de rotina.

Os Círculos de Cultura foram planejados, organizados e conduzidos pela pesquisadora, a qual foi identificada durante todo o processo educativo como coordenadora do Círculo ${ }^{9}$ A pesquisadora não tinha vínculo com as unidades de saúde, sendo uma pessoa externa aos locais onde a intervenção foi aplicada. Foram realizados seis círculos, quinzenalmente, com sessões de duas horas, durante um período de três meses, nos auditórios das unidades de saúde da área de residência do usuário.

Os Círculos se delinearam conforme as fases 8-10: a) investigação temática; b) escolha de temas geradores; c) problematização; d) desvelamento crítico. Em todas as fases, foram realizadas dinâmicas de acolhimento, com o objetivo de criar vínculo entre os pares.

O levantamento temático foi realizado pela coordenadora do círculo, a partir da observação de conversas e análise do Questionário de Atividades de Autocuidado com o Diabetes $(\mathrm{QAD})^{\mathbf{1 4}}$, que foi aplicado na primeira reunião com os participantes. O levantamento e a seleção dos temas se deram pela avaliação da não adesão às práticas de autocuidado no cotidiano dos participantes. Temas levantados pela coordenadora: alimentação, exercício físico, monitoramento glicêmico, cuidados com os pés e complicações do diabetes.

$\mathrm{Na}$ fase seguinte, escolha dos temas geradores, foi realizada, inicialmente, a apresentação e o acolhimento dos participantes. Depois, foi utilizada uma dinâmica ${ }^{15}$, na qual foram colocadas frases relacionadas com o convívio com o diabetes dentro de balões. A coordenadora pediu para que cada participante enchesse um balão, brincasse com ele por cinco minutos e refletisse sobre a seguinte pergunta: quais são os desafios em ter diabetes? Após brincarem com os balões, os participantes os estouraram e leram a frase, refletindo sobre a pergunta geradora lançada ao grupo, iniciando, desse modo, uma discussão entre os participantes sobre o diabetes, norteando a escolha dos temas 
geradores mais importantes para o grupo a serem dialogados: diabetes e complicações, alimentação, medicação e cuidados com os pés.

$\mathrm{Na}$ fase de problematização, esses temas geradores foram abordados por meio de fotos, desenhos, ilustrações e textos, com o objetivo de fomentar o diálogo entre os participantes. Além disso, foi estimulado o cuidado com os pés por meio da atividade prática do exame dos pés realizados entre os pares participantes do grupo. A fase de desvelamento crítico aconteceu concomitantemente com a fase de problematização, pois, ao dialogar sobre os temas geradores, analisando-os criticamente, os participantes tomaram consciência de sua realidade, sistematizando suas ideias ante o autocuidado no diabetes. Portanto, após a problematização, o grupo foi incentivado a expressar, por meio de falas, desenhos, palavras escritas em folha de papel, quais tomadas de decisões sobre o autocuidado com o diabetes passaram a ter, a partir das discussões no grupo.

Ainda foi realizado um círculo para o encerramento da intervenção em grupo, em que foi solicitado que o grupo fizesse a avaliação do método Círculos de Cultura. Para isso, foi elaborada pela coordenadora a dinâmica denominada Árvore do Autocuidado: utilizou-se uma árvore recortada em papel cartão, sendo entregue para os participantes uma ficha em forma de fruto, onde descreveram sentimentos, percepções sobre a intervenção educativa, abrindo para a discussão e questionamentos finais.

\section{Avaliação dos participantes}

A fim de comparar o resultado da intervenção para adoção de autocuidado entre os participantes do GC e do GI, foi aplicado o QAD ${ }^{14}$ e feita coleta da glicemia capilar, além de aferições do peso e altura para o cálculo do Índice de Massa Corporal (IMC), circunferência abdominal e pressão arterial sistólica e diastólica, para cada um dos participantes. Esse procedimento foi repetido após três meses, diferentemente do questionário sociodemográfico, aplicado apenas na reunião de apresentação do projeto.
O QAD, versão traduzida e validada para o português ${ }^{\mathbf{1 4}}$ do Summary of Diabetes Self-Care Activities Questionnaire (SDSCA) ${ }^{16}$, mede a aderência ao autocuidado dos usuários com Diabetes Mellitus tipo 2. Possui seis dimensões e 15 itens de avaliação do autocuidado com o diabetes: alimentação geral (com dois itens), alimentação específica (três itens), atividade física (dois itens), monitorização da glicemia (dois itens), cuidado com os pés (três itens) e 'uso da medicação' (três itens, utilizados de acordo com o esquema medicamentoso). Além disso, contêm outros três itens para a avaliação do tabagismo. As respostas variam de zero a sete dias, com os escores indicando as performances das atividades de autocuidado nos últimos sete dias da semana, sendo zero a situação menos desejável e sete a mais favorável. Apenas no segundo e no terceiro item da questão alimentação específica o escore zero indica a mais favorável; e sete, menos desejável.

A coleta da glicemia capilar em jejum de 8 horas a 12 horas ocorreu por meio de glicosímetro e fitas reagentes, o sangue total foi coletado de polpa digital por meio de lancetas estéreis 7 . A pressão arterial foi aferida por único examinador após cinco minutos de repouso, três medidas com intervalo de um minuto, em posição sentada, usando o método auscultatório por meio de esfigmomanômetro aneroide com estetoscópio, seguindo as orientações da VI Diretrizes Brasileiras de Hipertensão Arterial17. As medidas de peso e altura foram realizadas utilizando balança com estadiômetro acoplado da marca Welmy, com precisão de $0,1 \mathrm{~kg} \mathrm{e} 1 \mathrm{~cm}$, para peso e altura respectivamente. O IMC foi calculado pela relação do peso $(\mathrm{Kg})$ pela altura ao quadrado $\left(\mathrm{m}^{2}\right)^{\mathbf{1 8}}$. Por fim, a circunferência abdominal foi verificada por único aferidor na linha equidistante entre a margem mais inferior do arco costal e a crista ilíaca, medida por fita antropométrica inelástica e flexível com precisão de $0,1 \mathrm{~cm}^{\mathbf{1 8}}$.

\section{Análise estatística}

O teste de Kolmogorov-Smirnov foi aplicado para análise da distribuição de normalidade 
das variáveis. A diferença entre os GC e os GI na linha de base e a diferença em cada grupo antes e após a intervenção eram testadas utilizando o teste t-Student para duas amostras independentes ou amostras dependentes se a variável apresentasse distribuição normal com resultados em média e desvio padrão. Se a variável apresentasse distribuição não paramétricas, utilizava-se o teste de Mann-Whitney para duas amostras independentes ou o teste de Wilcoxon para comparação intragrupo antes e após o período de intervenção, com as variáveis apresentadas em medianas e quartis 25 e 75. A diferença entre proporções foi avaliada pelo teste do Qui-quadrado corrigido. Foram considerados estatisticamente significantes os valores de $\mathrm{p}<0,05$. O software SPSS 20.0 foi utilizado para as análises estatísticas.

\section{Resultados}

A população do estudo foi composta de 72 usuários com diabetes, sendo 41 do GI e 31 do GC. As mulheres corresponderam a 73,6\% dos participantes, a média de idade foi de 49,9 anos, e a escolaridade correspondeu a 6 anos de estudos completos. Não houve diferença estatisticamente significante entre os grupos GI e GC em relação ao gênero, à idade, ao estado civil, aos anos de estudo, aos trabalhadores ativos e à renda, conforme a tabela 1 abaixo.

Tabela 1. Características Sociodemográficas dos participantes dos Grupos Intervenção e Controle. Bragança, PA. 2017

\begin{tabular}{|c|c|c|c|}
\hline Características Sociodemográficas & Grupo Intervenção $(n=41)$ & Grupo Controle $(n=31)$ & $\mathrm{p}$ \\
\hline Gênero, n (\%) & & & $0,524^{\star}$ \\
\hline Homem & $12(29,3)$ & $7(22,6)$ & \\
\hline Mulher & $29(70,7)$ & $24(77,4)$ & \\
\hline Idade (anos) (média $\pm D P$ ) & $49,2 \pm 7,83$ & $51,0 \pm 7,30$ & $0,500^{\star \star}$ \\
\hline Estado civil, n (\%) & & & $0,616^{\star}$ \\
\hline Casado/união estável & $30(73,2)$ & $21(67,7)$ & \\
\hline Solteiro/Separado/Viúvo & $11(26,8)$ & $10(32,3)$ & \\
\hline Anos de estudo (mediana, Q25-Q75) & $6(4-8)$ & $6(3-9)$ & $0,895^{\star \star \star}$ \\
\hline Trabalhador ativo ou aposentado, $\mathrm{n}(\%)$ & & & $0,418^{\star}$ \\
\hline Ativo & $37(90,2)$ & $26(83,9)$ & \\
\hline Aposentado & $4(9,8)$ & $5(16,1)$ & \\
\hline Tipo de Atividade Ocupacional, n (\%) & & & $0,957^{\star}$ \\
\hline Prestador de serviços/comércio & $12(29,3)$ & $10(32,3)$ & \\
\hline Trabalhos domésticos & $21(51,2)$ & $16(51,6)$ & \\
\hline Agricultura/pesca & $8(19,5)$ & $5(16,1)$ & \\
\hline Renda (Reais) (mediana,Q25-Q75) & $937,00(0,00-1.171,25)$ & $468,00(0,00-937,00)$ & $0,266^{\star \star \star}$ \\
\hline
\end{tabular}

Analisando os parâmetros clínicos e a glicemia capilar na linha de base do estudo, observou-se não haver diferença estatisticamente significante entre os dois grupos (tabela 2). Os grupos apresentaram alta glicemia capilar em jejum, com média do GI de 211,6 mg/dL; e do GC de 174,2 $\mathrm{mg} / \mathrm{dL}$. A média do IMC indicou a existência de sobrepeso tanto no GI quanto no GC (tabela 2). 
Tabela 2. Comparação das variáveis clínicas e glicemia capilar entre os Grupos Intervenção e Controle na linha de base. Bragança, PA. 2017

\begin{tabular}{|c|c|c|c|}
\hline Variáveis & Grupo Intervenção $(n=41)$ & Grupo Controle $(n=31)$ & $p$ \\
\hline $\begin{array}{l}\text { Tempo de diagnóstico (anos) (mediana } \\
\text { Q25-Q75) }\end{array}$ & $2(1-5)$ & $3(1-7)$ & $0,330^{*}$ \\
\hline 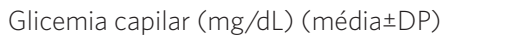 & $211,6 \pm 102,5$ & $174,2 \pm 67,4$ & $0,219^{\star \star}$ \\
\hline PAS (mmHg) (mediana, Q25-Q75) & $125(120-140)$ & $120(110-135)$ & $0,246^{\star}$ \\
\hline PAD (mmHg) (mediana, Q25-Q75) & $80(70-90)$ & $70(70-90)$ & $0,642^{\star}$ \\
\hline IMC $\left(\mathrm{Kg} / \mathrm{m}^{2}\right)$ (média $\left.\pm \mathrm{DP}\right)$ & $28,6 \pm 4,9$ & $27,3 \pm 5,7$ & $0,89^{\star \star}$ \\
\hline Circunferência abdominal (cm) (médiaさDP) & $96,3 \pm 9,5$ & $95,1 \pm 9,6$ & $0,887^{\star \star}$ \\
\hline
\end{tabular}

A avaliação do QAD na linha de base do estudo indicou que houve diferença estatisticamente significante entre os grupos em três itens: o GI comeu doce 1 dia na semana, enquanto o GC não comeu; o GI não examinou os sapatos antes de calçá-los, no entanto, o GC examinou os sapatos de 0 a 7 dias na semana; GI secou os espaços entre os dedos dos pés depois de lavá-los em 7 dias na semana, e GC, apenas 3 dias (tabela 3 ).

$\mathrm{O} Q A D$ possibilitou identificar que os itens 'tomou seus medicamentos do diabetes' e 'tomou o número indicado de comprimidos' foram os que tiveram maior adesão ao autocuidado nos dois grupos; e os itens 'praticou algum tipo de exercício físico específico' e 'avaliou o açúcar no sangue o número de vezes recomendado' tiveram a menor adesão entre os participantes dos grupos. Ressalta-se que, no item 'examinou dentro dos sapatos antes de calçá-los', os participantes do GI não tinham aderido a esse autocuidado em nenhum dia da semana. Adesão à insulina não pôde ser avaliada em virtude de não haver usuários, pois todos os participantes utilizavam medicação oral para o diabetes (tabela 3).

Tabela 3. Comparação dos itens do Questionário de Atividades de Autocuidado com o Diabetes (QAD) de acordo com o tempo de adesão nos Grupos Intervenção e Controle na linha de base. Bragança, PA. 2017

\begin{tabular}{|c|c|c|c|}
\hline \multirow[t]{2}{*}{ Itens do QAD } & Grupo Intervenção $(n=41)$ & Grupo Controle $(n=31)$ & \multirow[t]{2}{*}{$p^{\star}$} \\
\hline & Mediana (Q25-Q75) & Mediana (Q25-Q75) & \\
\hline Seguiu uma dieta saudável & $3,0(0,0-3,5)$ & $3,0(0,0-7,0)$ & 0,301 \\
\hline $\begin{array}{l}\text { Seguiu orientação alimentar de um profissional } \\
\text { de saúde }\end{array}$ & $3,0(0,0-3,0)$ & $2,0(0,0-5,0)$ & 0,647 \\
\hline $\begin{array}{l}\text { Comeu cinco ou mais porções de frutas e/ou } \\
\text { vegetais }\end{array}$ & $3,0(2,0-4,5)$ & $3,0(1,0-7,0)$ & 0,658 \\
\hline $\begin{array}{l}\text { Comeu carne vermelha, alimento com leite inte- } \\
\text { gral ou derivado }\end{array}$ & $3,0(2,0-5,0)$ & $3,0(2,0-5,0)$ & 0,903 \\
\hline Comeu doce & $1,0(0,0-1,0)$ & $0,0(0,0-1,0)$ & 0,034 \\
\hline $\begin{array}{l}\text { Realizou atividade física durante pelo menos } 30 \\
\text { minutos }\end{array}$ & $1,0(0,0-3,0)$ & $2,0(0,0-6,0)$ & 0,400 \\
\hline Praticou algum tipo de exercício físico & $0,0(0,0-0,0)$ & $0,0(0,0-0,0)$ & 0,101 \\
\hline Avaliou o açúcar do sangue & $1,0(0,0-1,0)$ & $0,0(0,0-1,0)$ & 0,903 \\
\hline Avaliou o açúcar do sangue recomendado & $0,0(0,0-0,0)$ & $0,0(0,0-0,0)$ & 0,324 \\
\hline Examinou os pés & $0,0(0,0-3,0)$ & $2,0(0,0-7,0)$ & 0,110 \\
\hline
\end{tabular}


Tabela 3. (cont.)

\begin{tabular}{lrrr}
\hline Itens do QAD & Grupo Intervenção (n=41) & Grupo Controle (n=31) & $\mathbf{p}^{\star}$ \\
\cline { 2 - 3 } & Mediana (Q25-Q75) & Mediana (Q25-Q75) & \\
\hline Examinou dentro dos sapatos antes de calçá-los & $0,0(0,0-0,0)$ & $0,0(0,0-7,0)$ & 0,042 \\
Secou os espaços dos dedos depois de lavá-los & $7,0(3,0-7,0)$ & $3,0(0,0-7,0)$ & 0,042 \\
Tomou seu medicamento de diabetes & $7,0(5,5-7,0)$ & $7,0(2,0-7,0)$ & 0,056 \\
Tomou sua injeção de insulina & $0,0(0,0-0,0)$ & $0,0(0,0-0,0)$ & 0,867 \\
Tomou o número indicado de comprimidos & $7,0(5,0-7,0)$ & $7,0(0,0-7,0)$ & 0,104 \\
\hline
\end{tabular}

*Teste Mann-Whitney.

Ao comparar as variáveis estudadas dos participantes do GI na linha de base e após três meses, foi observada redução da glicemia capilar em jejum de $211,6 \pm 102,5 \mathrm{mg} / \mathrm{dL}$ para $181,9 \pm 66,3 \mathrm{mg} / \mathrm{dL}$, pressão arterial sistólica de
125(120-140) mmHg para 120(117,5-130) $\mathrm{mmHg}$ e circunferência abdominal de $96,3 \pm 9,5$ para $95,5 \pm 9,1 \mathrm{~cm}$ (tabela 4). No GC não houve diferença estatisticamente significante nos parâmetros avaliados no período de estudo (tabela 4).

Tabela 4. Comparação das variáveis clínicas e glicemia capilar na linha de base e após três meses no Grupo Intervenção e no Grupo Controle. Bragança, PA. 2017

\begin{tabular}{|c|c|c|c|c|c|c|}
\hline \multirow[t]{2}{*}{ Variáveis } & \multicolumn{3}{|c|}{ Grupo Intervenção $(n=41)$} & \multicolumn{3}{|c|}{ Grupo Controle $(n=31)$} \\
\hline & Base & 3 meses & p & Base & 3 meses & p \\
\hline $\begin{array}{l}\text { Glicemia capilar (mg/dL) } \\
\text { (média } \pm \text { DP) }\end{array}$ & $211,6 \pm 102,5$ & $181,9 \pm 66,3$ & $0,020^{\star}$ & $174,2 \pm 67,4$ & $180 \pm 69,7$ & $0,685^{\star}$ \\
\hline $\begin{array}{l}\text { PAS (mmHg) (mediana, } \\
\text { Q25-Q75) }\end{array}$ & $125(120-140)$ & $120(117,5-130)$ & $0,045^{\star \star}$ & $120(110-135)$ & $120(110-130)$ & $0,323^{\star \star}$ \\
\hline $\begin{array}{l}\text { PAD (mmHg) (mediana, } \\
\text { Q25-Q75) }\end{array}$ & $80(70-90)$ & $80(70-90)$ & $0,968^{\star \star}$ & $70(70-90)$ & $80(70-90)$ & $0,693^{\star \star}$ \\
\hline $\begin{array}{l}\text { IMC }\left(\mathrm{Kg} / \mathrm{m}^{2}\right) \text { (mediana, } \\
\mathrm{Q} 25-\mathrm{Q} 75)\end{array}$ & $28(25-31,5)$ & $28(25-32)$ & $0,065^{\star \star}$ & $27(23-29)$ & $26(25-29)$ & $0,284^{\star \star}$ \\
\hline $\begin{array}{l}\text { Circunferência abdominal (cm) } \\
\text { (média } \pm \text { DP) }\end{array}$ & $96,3 \pm 9,5$ & $95,5 \pm 9,1$ & $0,001^{\star}$ & $95,1 \pm 9,6$ & $95,2 \pm 9,5$ & $0,557^{\star}$ \\
\hline
\end{tabular}

Fonte: Elaboração própria.

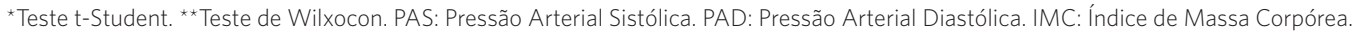

Os resultados comparativos intragrupos referentes ao QAD na linha de base e após três meses são apresentados na tabela 5 .

No GI, houve aumento estatisticamente significante no número de dias de adesão a dieta saudável, seguir orientação alimentar de um profissional da saúde, comer cinco ou mais porções de frutas e/ou vegetais, praticar algum tipo de exercício físico, avaliar o açúcar no sangue o número de vezes recomendado, examinar os pés, secar os espaços entre os dedos dos pés depois de lavá-los e tomar o número indicado de comprimidos. No grupo, houve redução no número de dias de consumo de carne vermelha, alimentos com leite integral e derivados e, ainda, doces (tabela 5).

No GC, houve aumento estatisticamente significante de dias de adesão apenas nas atividades tomar medicamentos do diabetes e tomar o número indicado de comprimidos, 
com diminuição de dias de adesão a dieta saudável, comer cinco ou mais porções de frutas e/ ou vegetais, comer carne vermelha, alimentos com leite integral ou derivados, praticar atividade física, examinar os pés e examinar dentro dos sapatos antes de calçá-los (tabela 5).

Tabela 5. Comparação dos itens do Questionário de Atividades de Autocuidado com o Diabetes (QAD) na linha de base e após três meses no Grupo Intervenção e no Grupo Controle. Bragança, PA. 2017

\begin{tabular}{|c|c|c|c|c|c|c|}
\hline \multirow[t]{2}{*}{ Itens do QAD } & \multicolumn{3}{|c|}{$\begin{array}{c}\text { Grupo Intervenção }(n=41) \\
\text { Mediana (Q25-Q75) }\end{array}$} & \multicolumn{3}{|c|}{$\begin{array}{l}\text { Grupo Controle }(n=31) \\
\text { Mediana }(Q 25-Q 75)\end{array}$} \\
\hline & Base & 3 meses & $\mathrm{p}^{\star}$ & Base & 3 meses & $\mathrm{p}^{\star}$ \\
\hline Seguiu uma dieta saudável & $3,0(0,0-3,5)$ & $4,0(3,0-5,0)$ & 0,006 & $3,0(0,0-7,0)$ & $2,0(0,0-3,0)$ & 0,008 \\
\hline $\begin{array}{l}\text { Seguiu orientação alimentar de } \\
\text { um profissional de saúde }\end{array}$ & $3,0(0,0-3,0)$ & $4,0(3,0-5,0)$ & 0,003 & $2,0(0,0-5,0)$ & $2,0(0,0-3,0)$ & 0,166 \\
\hline $\begin{array}{l}\text { Comeu cinco ou mais porções } \\
\text { de frutas e/ou vegetais }\end{array}$ & $3,0(2,0-4,5)$ & $4,0(3,0-5,0)$ & 0,003 & $3,0(1,0-7,0)$ & $2,0(2,0-3,0)$ & 0,017 \\
\hline $\begin{array}{l}\text { Comeu carne vermelha, ali- } \\
\text { mento com leite integral ou } \\
\text { derivado }\end{array}$ & $3,0(2,0-5,0)$ & $2,0(1,0-3,0)$ & 0,000 & $3,0(2,0-5,0)$ & $3,0(2,0-3,0)$ & 0,027 \\
\hline Comeu doce & $1,0(0,0-1,0)$ & $0,0(0,0-1,0)$ & 0,008 & $0,0(0,0-1,0)$ & $1,0(0,0-1,0)$ & 0,387 \\
\hline $\begin{array}{l}\text { Realizou atividade física duran- } \\
\text { te pelo menos } 30 \text { minutos }\end{array}$ & $1,0(0,0-3,0)$ & $2,0(0,0-3,0)$ & 0,847 & $2,0(0,0-6,0)$ & $0,0(0,0-3,0)$ & 0,002 \\
\hline $\begin{array}{l}\text { Praticou algum tipo de exercí- } \\
\text { cio físico }\end{array}$ & $0,0(0,0-0,0)$ & $0,0(0,0-2,6)^{\star \star}$ & 0,005 & $0,0(0,0-0,0)$ & $0,0(0,0-0,0)$ & 0,180 \\
\hline Avaliou o açúcar do sangue & $1,0(0,0-1,0)$ & $1,0(0,0-1,5)$ & 0,086 & $0,0(0,0-1,0)$ & $0,0(0,0-1,0)$ & 0,827 \\
\hline $\begin{array}{l}\text { Avaliou o açúcar do sangue o } \\
\text { recomendado }\end{array}$ & $0,0(0,0-0,0)$ & $1,0(0,0-1,5)$ & 0,001 & $0,0(0,0-0,0)$ & $0,0(0,0-1,0)$ & 0,090 \\
\hline Examinou os pés & $0,0(0,0-3,0)$ & $5,0(3,0-7,0)$ & 0,000 & $2,0(0,0-7,0)$ & $0,0(0,0-3,0)$ & 0,000 \\
\hline $\begin{array}{l}\text { Examinou dentro dos sapatos } \\
\text { antes de calçá-los }\end{array}$ & $0,0(0,0-0,0)$ & $0,0(0,0-2,0)$ & 0,970 & $0,0(0,0-7,0)$ & $0,0(0,0-0,0)$ & 0,001 \\
\hline $\begin{array}{l}\text { Secou os espaços dos dedos } \\
\text { depois de lavá-los }\end{array}$ & $7,0(3,0-7,0)$ & $7,0(6,0-7,0)$ & 0,009 & $3,0(0,0-7,0)$ & $1,0(0,0-5,0)$ & 0,241 \\
\hline $\begin{array}{l}\text { Tomou seu medicamento de } \\
\text { diabetes }\end{array}$ & $7,0(5,5-7,0)$ & $7,0(7,0-7,0)$ & 0,106 & $7,0(2,0-7,0)$ & $7,0(7,0-7,0)$ & 0,004 \\
\hline Tomou sua injeção de insulina & $0,0(0,0-0,0)$ & $0,0(0,0-0,0)$ & 0,655 & $0,0(0,0-0,0)$ & $0,0(0,0-0,0)$ & 0,317 \\
\hline $\begin{array}{l}\text { Tomou o número indicado de } \\
\text { comprimidos }\end{array}$ & $7,0(5,0-7,0)$ & $7,0(7,0-7,0)$ & 0,047 & $7,0(0,0-7,0)$ & $7,0(6,0-7,0)$ & 0,007 \\
\hline
\end{tabular}

Fonte: Elaboração Própria.

*Teste de Wilcoxon. Percentil 25-Percentil 80.

Não houve diferença estatisticamente significante entre os GI e os GC na linha de base em relação ao tabagismo. Verificou-se que 37 (90,2\%) dos participantes do GI e que 30 (96,8\%) do GC não usaram cigarro nos últimos sete dias ( $\mathrm{p}=0,280)$; e, destes, 4 usuários do GI
(9,8\%) e 1 usuário do GC (3,2\%) fumaram no dia da entrevista, enquanto $20(48,8 \%)$ participantes do GI e $18(58,1 \%)$ do GC relataram que nunca fumaram $(\mathrm{p}=0,435)$. Após três meses do estudo, não houve mudança estatisticamente significante no hábito de fumar, sendo que 
39 (95,1\%) usuários do GI e 30 participantes (96,8\%) do GC mantiveram o não uso nos últimos sete dias ( $\mathrm{p}=0,728)$, três participantes $(7,3 \%)$ do GI e um do GC $(3,2 \%)$ relataram o consumo de cigarro no dia. Portanto, permaneceram 20 (48,8\%) no GI e 18 (58,1\%) no GC que nunca fumaram $(\mathrm{p}=0,603)$.

\section{Discussão}

A adoção de práticas de autocuidado pelo paciente pode ser influenciada por vários fatores, como a experiência de vida, os aspectos sociais e culturais, presença de sentimentos depressivos, desmotivação, sendo exigido do paciente, no que concerne ao autocuidado, tomadas de decisões, controle do comportamento e aquisição de conhecimentos e habilidades ${ }^{\mathbf{1 9}, 20}$. Por isso, é importante que o profissional de saúde reconheça os fatores que levam ou não a uma adoção de autocuidado, evitando atitudes prescritivas ao abordar os pacientes com diabetes, empregando práticas motivacionais e comportamentais que contribuam para o empoderamento e para a autonomia dos pacientes perante o autocuidado ${ }^{21,22}$.

Os Círculos de Cultura desenvolvidos neste estudo, como educação problematizadora em saúde, ao valorizar os participantes, seus saberes, suas experiências, construiu um processo educativo em que a pesquisadora não foi a detentora do saber, mas uma facilitadora do processo de ensino-aprendizagem ${ }^{11}$. Para Heidemann et al. ${ }^{23(6)}$, o método freireano promove

espaços de encontro entre as pessoas, rompendo com as barreiras hierárquicas implicadas na lógica biomédica, democratizando o saber em saúde, valorizando os cotidianos, as culturas e as formas de pensar e viver das famílias, grupos e coletividades.

Para Santos et al. ${ }^{\mathbf{2 4}}$, os Círculos de Cultura, enquanto prática educativa participativa, que promove a troca de diálogo, conhecimento e experiências, geram autonomia e transformações no automanejo do diabetes, na aceitação dessa condição crônica e na responsabilidade do paciente referentes a sua saúde.

Para Paulo Freire"1, as pessoas aprendem o que é significativo para elas, portanto, a abordagem educativa deve partir do conhecimento do usuário, do seu contexto de vida, dos obstáculos vividos, sendo importante que o profissional de saúde respeite e valorize o saber da pessoa, que será primordial para reflexões e construção do entendimento sobre $o$ autocuidado com o diabetes.

Referente às atividades de autocuidado analisadas neste estudo, percebeu-se que o uso da medicação foi a atividade de maior realização pelos participantes, o que corrobora os estudos de Coelho et al.25. Para esses autores, o tratamento para pessoas com diabetes consiste em adoção de mudanças no estilo de vida, em que os pacientes podem ou não fazer uso de medicação. No entanto, quando a medicação faz parte do controle do diabetes, os pacientes têm melhor adesão a esse autocuidado do que ao tratamento não farmacológico, pois o medicamento, ao ser fornecido pelas unidades de saúde, não gera gastos financeiros na maioria das vezes, estimulando assim a terapia medicamentosa como autocuidado adotado pelos pacientes ${ }^{26}$. Esse fato foi observado nos diálogos dos participantes do presente estudo ao considerarem a terapia medicamentosa como a atividade de maior importância para controlarem o diabetes.

A alimentação saudável foi uma das atividades que apresentaram menores adoções de autocuidado pelos participantes. As barreiras por eles expressas se relacionavam com questões culturais, muitas vezes adquiridas na infância, como o consumo da farinha, do 'xibé' (mistura de água com farinha de mandioca, muito utilizada no acompanhamento com peixes), muito frequente no município de Bragança e no Pará, e dificultando a mudança no hábito alimentar dos pacientes ${ }^{26}$. Para Salci et al. ${ }^{27}$, a atenção à saúde deve dar destaque aos aspectos culturais do indivíduo, buscando compreender sua visão de mundo, reconhecendo 
o contexto social, familiar e financeiro vivido pelo paciente, visto que esses fatores podem influenciar ou não em hábitos de alimentação saudável da população ${ }^{28}$.

A alimentação está relacionada diretamente com alguns fatores que interferem na prevenção ou no controle do diabetes e seus agravos, sendo necessário promover educação em saúde com enfoque nos hábitos alimentares que, na maioria dos casos, se modificados, têm potencial para evitar e/ou retardar as complicações dessa doença ${ }^{29,30}$. Portanto, o diálogo construído nos grupos sobre a alimentação saudável, problematizando e desvelando as barreiras culturais e financeiras na prática do autocuidado, resultou que os participantes do GI, mais do que os do GC, passassem a seguir com maior frequência uma dieta mais saudável, consumindo mais frutas, vegetais, além de ter diminuído o consumo de gorduras e doces.

Sobre o exercício físico, não havia adesão a esse autocuidado no início do estudo. A não adesão pode estar relacionada com a falta de tempo e com a rotina de trabalho dos participantes, já que, no entendimento deles, suas funções ocupacionais exercendo atividades domésticas em seu domicílio já são consideradas uma forma de exercitar-se. No entanto, é importante mencionar que os serviços domésticos são considerados atividades físicas de leve intensidade ${ }^{31}$, sendo que as recomendações para pacientes com diabetes é a prática de exercício físico de intensidade moderada, realizada por 150 minutos, cinco vezes na semana ou, então, exercício físico de alta intensidade, com duração de 75 minutos, três vezes por semana ${ }^{13}$. Outro ponto importante a destacar é que no município onde o estudo foi realizado, apesar da existência da Academia da Saúde, programa do Ministério da Saúde que tem como um dos objetivos específicos o aumento de atividade física da população, principalmente para a prevenção e o controle das doenças crônicas não transmissíveis ${ }^{32}$, observou-se que muitos pacientes nunca usufruíram desse serviço de saúde, alguns por desconhecerem o programa, outros por não se sentirem motivados ao deslocamento de suas casas e à participação nas ações de saúde, o que remete à reflexão de que esses pacientes ainda não conseguiram se ver como protagonistas nesse processo de autocuidado. Nesse sentido, é fundamental que os profissionais de saúde percebam as dificuldades e as barreiras apresentadas pelos usuários, e busquem criar estratégias que contribuam para sensibilização da importância da adoção do autocuidado ${ }^{33}$. Desse modo, conhecer e refletir sobre os benefícios da atividade física no GI melhoraram a prática do autocuidado. A prática regular do exercício físico é fundamental no tratamento do diabetes, que além de melhorar o controle glicêmico, diminui os fatores de risco para a doença coronariana, contribui para a perda de peso e melhora o bem-estar das pessoas com diabetes ${ }^{30}$.

Acerca do monitoramento glicêmico, pouca adesão foi verificada nesse autocuidado, tendo como motivo a falta dos insumos nas unidades de saúde, situação existente há alguns meses. No estudo de Coelho et al. ${ }^{25}$, também se verificou a mesma dificuldade de acesso aos insumos para a realização do monitoramento glicêmico por usuários em Unidades Básicas de Saúde. Outro fator importante relacionou-se com a dificuldade financeira em comprar o glicosímetro e as fitas reagentes. Ressalta-se que, no presente estudo, as médias das rendas dos participantes foram de $\mathrm{R} \$ 937,00$ para o GI e de $\mathrm{R} \$ 468,00$ no GC, o que torna a compra desses insumos como não sendo prioritária. $\mathrm{O}$ monitoramento da glicemia é importante para que o participante avalie a resposta individual à terapia, pois ele pode observar se as metas glicêmicas recomendadas estão sendo alcançadas ${ }^{7}$. Então, é importante que os usuários compreendam o que é meta glicêmica, já que no GI havia desconhecimento desse termo. Assim, foi debatido sobre glicemia, meta glicêmica e a importância do paciente conhecer esse parâmetro e realizar o monitoramento; e apesar da falta dos insumos nas unidades de saúde e dificuldades financeiras dos participantes, eles compreenderam a importância do autocuidado, já que se constatou que os 
participantes do GI aumentaram o monitoramento da glicemia após a intervenção do círculo de cultura.

$\mathrm{O}$ autocuidado com os pés também apresentou baixa execução pelos participantes, principalmente a prática de examinar os sapatos antes de calçá-los. Os usuários, que têm o costume do uso de chinelas e sandálias abertas, entendiam que os calçados abertos não precisariam ser inspecionados, demonstrando a falta de entendimento sobre as recomendações sobre os cuidados com os pés para pessoas com diabetes ${ }^{34}$. Os Agentes Comunitários de Saúde que participaram de alguns círculos relataram que a orientação sobre os pés não faz parte de sua rotina de trabalho. $\mathrm{O}$ achado corrobora estudo realizado por Neta et al. ${ }^{35}$, no qual foi observado que a maioria dos participantes nunca tinha recebido orientação do profissional de enfermagem sobre os cuidados com os sapatos antes de calçá-los, contrariando uma das funções do profissional, que é ajudar o paciente no autocuidado que promova a prevenção ao pé diabético.

Ademais, mesmo com expansão das ofertas de serviços saúde e de maior ênfase no cuidado com as doenças crônicas, o Ministério da Saúde enfoca a ocorrência frequente de complicações com os pés causados pelo diabetes, algo que poderia ser evitado com o bom manejo das equipes de saúde para essa complicação ${ }^{36}$, sendo importante a abordagem educativa para a prevenção das complicações dos pés, enfatizando a relevância do cuidado diário com eles?

O profissional de saúde que respeita os saberes dos usuários permite que eles possam relacionar suas experiências de vida e comunitária com os conteúdos técnicos-científicos repassados pelo profissional, permitindo-lhes que se tornem sujeitos críticos e problematizadores a caminho de uma autonomia tão necessária que eles ainda não possuem ${ }^{37}$.

Quando se analisou o GC, observou-se que houve diminuição de práticas saudáveis de alimentação, com menos consumo de vegetais e frutas, aumento no consumo de doce, redução de prática de atividade física e exame dos pés, além do aumento da glicemia capilar e da circunferência abdominal. Desse modo, percebeu-se que os Círculos de Cultura como intervenção educativa para pacientes com Diabetes Mellitus foram eficientes na melhor adoção ao autocuidado dos participantes do GI, na redução da glicemia capilar em jejum, pressão arterial sistólica e circunferência abdominal, assim como no estudo de Pereira et al. ${ }^{38}$. Para esses autores, o diálogo vivenciado, os relatos de experiências compartilhadas e as reflexões sobre as atitudes perante a doença são primordiais para um melhor conhecimento do diabetes, que levam os pacientes a compreenderem a sua condição de portador, desvelando mudança de hábitos, com adoção de práticas de autocuidado, principalmente na alimentação, na atividade física e nos cuidados com os pés ${ }^{38}$.

\section{Considerações finais}

Os círculos de cultura foram essenciais em mudanças de atitudes dos participantes, que puderam dialogar sobre os temas geradores, problematizando-os e os desvelando, compartilhando saberes, fortalecendo a autonomia dos envolvidos, possibilitando melhor compreensão sobre o autocuidado no Diabetes Mellitus, refletidos nos resultados obtidos.

Mesmo com o apoio e a integração construídos entre a pesquisadora e os participantes por meio dos diálogos, constatou-se que ainda são frequentes práticas educativas verticalizadas no município onde o estudo ocorreu. $\mathrm{O}$ cuidado prestado às pessoas com diabetes ainda se apresenta pautado no modelo centrado no profissional de saúde, em que não há criação de vínculo, não havendo acolhimento e humanização na assistência prestada aos pacientes, que não permite a integralidade preconizada para que o paciente seja o protagonista do processo 39 .

Uma inversão desse modelo passa por múltiplas dimensões, da gestão ao serviço, considerando o usuário como protagonista 
do cuidado. Isso implicaria, por exemplo, a adoção de um processo de formação em educação permanente em saúde no município, reconhecendo práticas e saberes e, com eles, provocando a produção de novos sentidos no fazer saúde. Assim, a perspectiva da formação deve ser vivenciada, ao invés de momentos específicos e longe dos usuários, no encontro diário entre profissionais de saúde e entre estes e os usuários, promovendo um processo que traz consigo os atributos da liberdade, criação e transformação, invertendo para o modelo centrado no usuário ${ }^{40}$.

Desenvolver este estudo, portanto, foi relevante para os atores envolvidos, para a academia e para o município, sugerindo-se assim a elaboração de um plano de educação permanente em saúde que englobe atividades direcionadas para os pacientes, com ênfase em metodologias problematizadoras, como os Círculos de Cultura.

\section{Colaboradores}

Correa ST (0000-0003-0509-0829)* contribuiu para a concepção, planejamento, análise e interpretação dos dados; elaboração do rascunho e revisão crítica do conteúdo e aprovação da versão final do manuscrito. Castelo-Branco S (0000-0001-6283-0446)* contribuiu para a concepção, planejamento, análise, interpretação dos dados; revisão crítica do conteúdo; aprovação da versão final do manuscrito.

\section{Referências}

1. International Diabetes Federation. IDF Diabetes Atlas. 7. ed. Brussels: IDF; 2015.

2. Organização Pan-Americana da Saúde. Doenças crônicas-degenerativas e obesidade: estratégia mundial sobre alimentação saudável, atividade física e saúde. Brasília, DF: OPAS; 2003.

3. Instituto Brasileiro de Geografia e Estatística. Pesquisa Nacional de Saúde 2013. Percepção do Estado de Saúde, Estilos de Vida e Doenças Crônicas. Rio de Janeiro: IBGE; 2014.

4. Brasil. Ministério da Saúde. VIGITEL 2016: Vigilância de Fatores de Risco e Proteção para Doenças Crônicas por Inquérito Telefônico. Brasília, DF: MS; 2017.

5. Organização Mundial da Saúde. Cuidados Inovadores para Condições Crônicas: Componentes Estruturais de Ação: Relatório Mundial. Brasília, DF: OMS; 2003.
${ }^{*}$ Orcid (Open Researcher and Contributor ID). 
6. Merhy EE. Saúde: cartografia do trabalho vivo em ato. São Paulo: Hucitec; 2002.

7. American Diabetes Association. Standards of Medical Care in Diabetes - 2015. Diab. Care [internet]. 2015 [acesso em 2016 out 13]; 38(supl1):1-99. Disponível em: http://www.diabetes.teithe.gr/UsersFiles/ entypa/Standards\%20of\%20medical\%20care\%20 in\%20diabetes\%202015.pdf.

8. Heidemann ITSB, Prado ML, Reibnitz KS. Curso de Especialização em Linhas de Cuidados em Enfermagem. Modulo III: Processo Educativo em Saúde. Florianópolis: UFSC; 2013.

9. Brandão CR. O que é o Método Paulo Freire. São Paulo: Brasiliense; 2013.

10. Freire P. Educação como Prática da Liberdade. Rio de Janeiro: Paz \& Terra; 1967.

11. Freire P. Pedagogia da Autonomia: saberes necessários à prática educativa. 47. ed. Rio de Janeiro: Paz \& Terra; 2013.

12. Brasil. Ministério da Saúde. Portaria n ${ }^{\circ}$ 2.761, de 19 de novembro de 2013. Institui a Política Nacional de Educação Popular em Saúde no Âmbito do Sistema Único de Saúde (PNEPSSUS). Diário Oficial da União. 19 Nov 2013.

13. Sociedade Brasileira de Diabetes. Diretrizes da Sociedade Brasileira de Diabetes: 2014-2015. Sociedade Brasileira de Diabetes. São Paulo: AC Farmacêutica; 2015.

14. Michels MJ, Coral MHC, Sakae TM, et al. Questionário de Atividades de Autocuidado com o Diabetes: tradução, adaptação e avaliação das propriedades psicométricas. Arq. Bras. Endocrinol. Metab. 2010; 54(7):644-651.

15. Simionato RGB. Dinâmicas de grupo para treinamento motivacional. 8. ed. Campinas: Papirus; 2012.

16. Toobert DJ, Hampson SE, Glasgow RE. The summary of diabetes self-care activities measure: results from 7 studies and revised scale. Diabetes Care. 2000; 23(7):943-50.

17. Sociedade Brasileira de Cardiologia / Sociedade Brasileira de Hipertensão / Sociedade Brasileira de Nefrologia. VI Diretrizes Brasileiras de Hipertensão. Arq. Bras. Cardiol.; 2010.

18. World Health Organization. Obesity: preventing and managing the global epidemic. Report of a World Health Organization Consultation. Geneva: WH; 2000.

19. Orem DE. A Teoria Geral de Enfermagem de Orem. Porto Alegre: Artes Médicas; 1993.

20. Oliveira NF, Souza MCBM, Zanetti ML, et al. Diabetes Mellitus: desafios relacionados ao autocuidado abordados em grupo de apoio psicológico. Rev Bras Enferm. 2011; 64(2):301-7.

21. Anderson RM, Funnel MM, Burkhart N, et al. 1001 Tip for behavior change in diabetes education. Virgínia: American Diabetes Association; 2002.

22. Baquedano IR, Santos MA, Martins TA, et al. Autocuidado de pessoas com diabetes mellitus atendidos em serviço de urgência no México. Revista Latino-Am Enfermagem [internet]. 2010 [acesso em 2017 jun 16]; 18:(6):1-9. Disponível em: http://www.scielo. br/pdf/rlae/v18n6/pt_21.pdf.

23. Heidmann ITSB, Dalmoni IS, Rumon PCF, et al. Reflexões sobre o Itinerário de Pesquisa de Paulo Freire: contribuições para a saúde. Texto Contexto Enferm. [internet]. 2017 [acesso em 2019 set 10]; 26(4):1-8. Disponível em: http://www.scielo.br/pdf/ tce/v26n4/0104-0707-tce-26-04-e0680017.pdf.

24. Santos AL, Cecilio HPM, Marcon SS. Percepção de pessoas com diabetes mellitus tipo 2 acerca de um processo de educação para a saúde. Rev Rene. [internet]. 2015 [acesso em 2019 set 10]; 16(4):522-31. Disponível em: http://www.redalyc.org/articulo. oa?id=324041519009.

25. Coelho ACM, Villas-Boas LC, Gomides DDS, et al. Atividades de autocuidado e suas relações com o con- 
trole metabólico e clínico das pessoas com diabetes mellitus. Texto Contexto Enferm. 2015; 24(3):697705.

26. Faria HTG, Santos MA, Arrelias CCA, et al. Adesão ao tratamento em diabetes mellitus em unidades da Estratégia Saúde da Família. Rev Esc Enferm USP. 2014; 48(2):257-63.

27. Salci MA, Maceno P, Rozza SG, et al. Educação em saúde e suas perspectivas teóricas: algumas reflexes. Texto Contexto Enferm. 2013; 22(1):224-30.

28. Brasil. Ministério da Saúde. Guia Alimentar para a população brasileira. Brasília, DF: MS; 2014.

29. World Health Organization. Global Status Report on Noncommunicable Diseases 2014. Geneva: WHO; 2014.

30. Brasil. Ministério da Saúde. Estratégias para o cuidado da pessoa com doença crônica: diabetes mellitus caderno ${ }^{\circ}{ }^{36}$. Brasília, DF: MS; 2014.

31. Caspersen CJ. Physical activity, exercise and physical fitness: definitions and distinctions for health related research. Public Health Reports. 1985; 100(2):172-179.

32. Brasil. Ministério da Saúde. Portaria no 2.681, de 7 de novembro de 2013. Redefine o Programa Academia da Saúde no âmbito do Sistema Único da Saúde (SUS). Diário Oficial da União. 7 Nov 2013.

33. Silva MAV, Gouvêa GR, Claro AFB, et al. Impacto da ativação da intenção na prática da atividade física em diabético tipo II: ensaio clínico randomizado. Ciênc. Saúde Colet. 2015; 20(3):875-886.

34. International Diabetes Federation. IDF: Clinical Prac- tice Recommendations on the Diabetes Foot 2017: a guide for healthcare professional. Bruxelas: International Diabetes Federation; 2017.

35. Neta DSR, Silva ARVD, Silva GRF. Adesão das pessoas com diabetes mellitus ao autocuidado com os pés. Rev. Bras Enferm. 2015; 68(1):111-6.

36. Brasil. Ministério da Saúde, Secretaria de Atenção à Saúde, Departamento de Atenção Básica. Manual do pé diabético: estratégias para o cuidado da pessoa crônica. Brasília, DF: MS; 2016.

37. Paula M. Estudo Sobre a Pedagogia da Autonomia de Paulo Freire [internet]. Porto Alegre: Editora Fi; 2016. [acesso em 2018 dez 13]. Disponível em: https://3c290742-53df-4d6f-b12f-6b135a606bc7.filesusr.com/ugd/48d206_8555bcd62f3a407a90d49 b00a9bc372c.pdf.

38. Pereira DA, Costa NMSC, Souza ALL, et al. Efeito de intervenção educativa sobre o conhecimento da doença em pacientes com diabetes mellitus. Rev. Latino-Am. Enfermagem. 2012; 20(3):1-8.

39. Bastos LS, Assis MMA, Nascimento MAA, et al. Construção da integralidade no cuidar de pessoas com diabetes mellitus em um centro de saúde em Feira de Santana (BA). Ciênc. Saúde Colet. 2011; 16(1):14171426.

40. Merhy EE. Em busca do tempo perdido: A micropolítica do trabalho vivo em saúde. In: Merhy EE, Onocko R, organizadores. Agir em Saúde: Um desafio para o público. São Paulo: Hucitec; 2007. p. 71-112.

Recebido em 12/03/2019

Aprovado em 30/09/2019

Conflito de interesses: inexistente

Suporte financeiro: não houve 